\title{
Retroperitoneal single-site robot-assisted partial nephrectomy using Lapsingle Vision advanced access platform: initial three case reports
}

\author{
Hyung Ho Lee ${ }^{1}$, Young Eun Yoon ${ }^{2}$, Young Sig Kim ${ }^{3}$, Joon Chae $\mathrm{Na}^{4}$, Koon Ho Rha ${ }^{4}$, Woong Kyu Han \\ Dae Keun Kim $^{5}$ \\ ${ }^{1}$ Department of Urology, Center for Urologic Cancer, National Cancer Center, Gyeonggi-do, Republic of Korea; ${ }^{2}$ Department of Urology, Hanyang \\ University College of Medicine, Seoul, Republic of Korea; ${ }^{3}$ Department of Urology, National Health Insurance Service Ilsan Hospital, Gyeonggi- \\ do, Republic of Korea; ${ }^{4}$ Department of Urology, Urological Science Institute, Yonsei University College of Medicine, Seoul, Republic of Korea; \\ ${ }^{5}$ Department of Urology, CHA Fertility Center Seoul Station, CHA University School of Medicine, Seoul, Republic of Korea \\ Correspondence to: Dae Keun Kim. Department of Urology, CHA Fertility Center Seoul Station, CHA University School of Medicine, 416, Hangang- \\ daero, Jung-gu, Seoul 04367, Republic of Korea. Email: kdg070723@gmail.com.
}

\begin{abstract}
Robot-assisted partial nephrectomy is currently the standard for treatment of small renal mass. Recently, robot-assisted single site surgery has been introduced. However, there have been few reports of retroperitoneal approaches. Herein, we report initial case series of retroperitoneal single-site robot-assisted partial nephrectomy using the da Vinci Xi surgical system using the Lapsingle Vision advanced access platform. Three patients have undergone retroperitoneal single-site robot-assisted partial nephrectomy due to incidental finding of renal mass. Operation duration, estimated blood loss, warm ischemia time, estimated glomerular filtration rate (eGFR) change, and complication were evaluated. Renal cell carcinoma of the two clear cell type and one chromophobe was diagnosed based on the pathological examination. Initial two cases were successfully completed with minimal bleeding and warm ischemic time within 25 minutes. The last 3rd case has been converted to multiport operation due to limited retroperitoneal space and difficulty in managing upper pole renal mass. Retroperitoneal single-site robot-assisted partial nephrectomy is a feasible treatment modality for treatment of posterior or lateral renal masses. Additional cases are needed to confirm the safety and efficacy of this technique.
\end{abstract}

Keywords: Kidney cancer; retroperitoneal; nephrectomy; robotics; single site case reports

Submitted Dec 17, 2019. Accepted for publication Jan 08, 2020.

doi: $10.21037 /$ tau.2020.01.14

View this article at: http://dx.doi.org/10.21037/tau.2020.01.14

\section{Introduction}

Urologic surgery is undergoing an exciting period of growth, not least because of introduction of the da Vinci surgical system, and clinical application of laparoendoscopic single-site surgery (LESS). According to the literature, LESS is technically feasible and safe for treatment of various urologic diseases, resulting in better cosmetic outcomes, lower postoperative analgesic requirements, and faster convalescence than is available with conventional laparoscopy $(1,2)$. Nevertheless, the initial da Vinci system (Intuitive Surgical Inc, Sunnyvale, CA, USA) was not designed for use in single-site surgery. Intuitive Surgical Inc. developed a novel set of single-site instruments and accessories specifically designed for single-site surgery in an attempt to overcome the current limitations $(3,4)$. LESS has logically evolved to robot-assisted-LESS (R-LESS) because of the benefits of automatic re-association of the surgeon's hands with the instrument, ergonomics and 3D vision. Therefore, R-LESS procedures have become more common recently.

By contrast with the recent decline in the use of LESS (5), robotic-assistance appears to improve ergonomics, therefore 

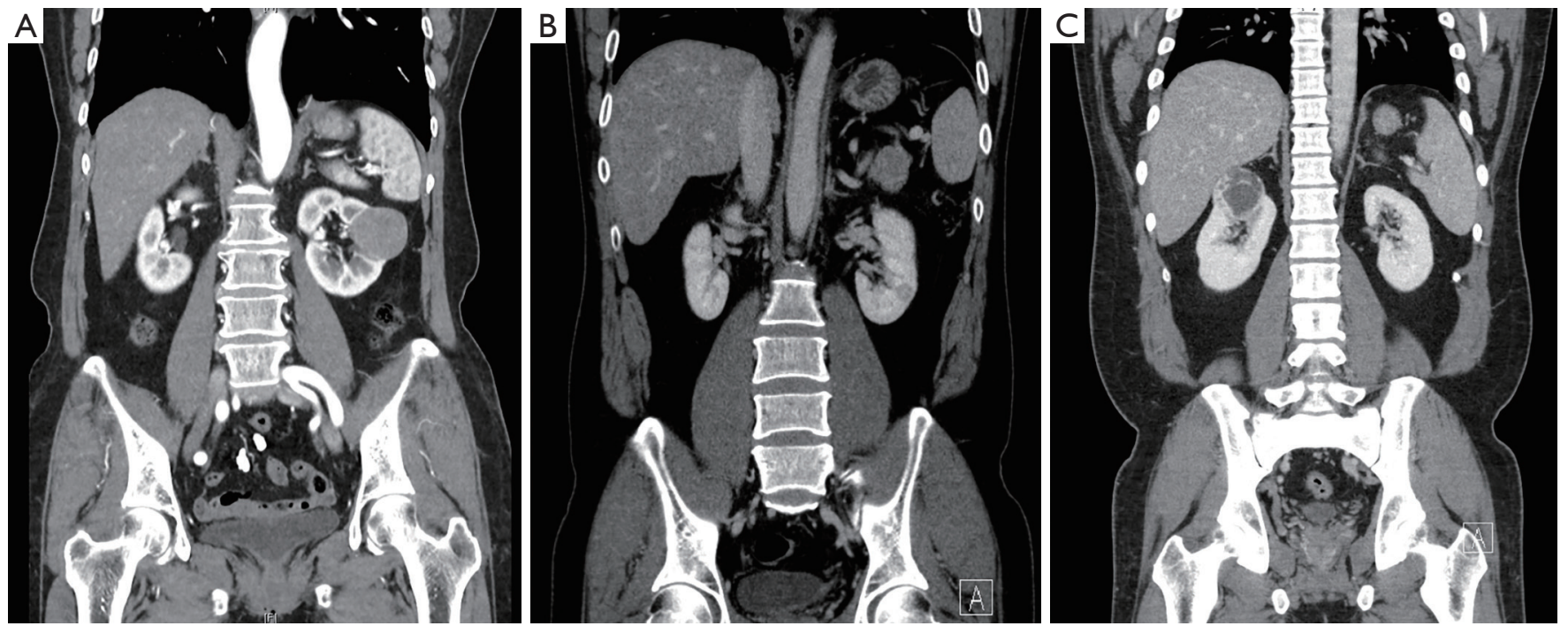

Figure 1 Computed tomography imaging of the tumors in three cases, 1 (A), 2 (B), and 3 (C).

Table 1 Demographic and clinical characteristics of the patient

\begin{tabular}{lccc}
\hline Characteristic & Case 1 & Case 2 & Case 3 \\
\hline Age (years) & 66 & 51 & 38 \\
Sex & Female & Male & Male \\
Body mass & 24.56 & 26.76 & 33.86 \\
index (kg/m ${ }^{2}$ ) & & & \\
ASA classification & 2 & 2 & 2 \\
Comorbidities & None & HBV & Hypertension, DM \\
Previous surgery & None & None & None \\
Side & Left & Left & Right \\
Tumor size (cm) & $4.5 \times 4.0$ & $1.2 \times 1.0$ & $5.0 \times 4.0$ \\
Location & Lateral, & Anterior, & Posterior, \\
& mid-pole & lower & upper \\
RENAL score & 10 & 7 & 10 \\
\hline
\end{tabular}

ASA, American Society of Anesthesiologists; HBV, hepatitis B virus; DM, diabetes mellitus; RENAL, radius exophytic/ endophytic nearness to collecting system or sinus anterior/ posterior location relative to polar lines.

initiating a new era for LESS. However, the initial da Vinci platform was not specifically designed for this purpose. The introduction of the novel da Vinci single-site platform and its use in urology has been previously reported $(6,7)$. Several centers reported their techniques and initial outcomes for R-LESS partial nephrectomy $(2,8)$. However, there have been no reports of retroperitoneal approaches using the da
Vinci Xi surgical system.

In this case series, we described the advantage and disadvantage of retroperitoneal single-site robot-assisted partial nephrectomy (R-SSRPN) using the da Vinci Xi surgical system.

\section{Case presentation}

\section{Case 1}

A 66-year-old woman was diagnosed with a 4.5 -cm lateral renal mass located in the left mid-pole kidney (Figure 1A). The da Vinci Xi surgical system (Intuitive Surgical, Sunnyvale, CA, USA) was used to perform R-SSRPN. Patient has provided informed consent for publication of this case.

The left-sided mid-pole lateral $4.5-\mathrm{cm}$ renal mass (cT1b) was successfully removed using R-SSRPN without any additional port. The demographic and clinical characteristics of a patient are presented in Table 1. The patient body mass index (BMI) was $24.56 \mathrm{~kg} / \mathrm{m}^{2}$. The total operative time was 100 minutes. Console and docking time were 80 and 9 minutes, respectively. The estimated blood loss was $30 \mathrm{~mL}$. The warm ischemia time was 23 minutes. No intraoperative and neither postoperative complications occurred (Clavien-Dindo 0). Hospital duration was 3 days. Preoperative estimated glomerular filtration rate (eGFR) was $75.20 \mathrm{~mL} / \mathrm{min} / 1.73 \mathrm{~m}^{2}$ and postoperative 1-week eGFR was $67.40 \mathrm{~mL} / \mathrm{min} / 1.73 \mathrm{~m}^{2}$. The pathology result revealed 
pT1bN0M0 chromophobe renal cell carcinoma (ISUP grade II) with a negative surgical margin (Table 2).

\section{Case 2}

A 51-year-old man had hypertension and diagnosed with a $1.3-\mathrm{cm}$ lateral renal mass in the left lower-pole kidney (Figure 1B). The patient BMI was 26.73 (Table 1). The leftsided lower-pole lateral renal mass was successfully removed by R-SSRPN without any additional port. Total operative time was 118 minutes. The estimated blood loss was minimal $(5 \mathrm{~mL})$. The warm ischemia time was 21 minutes. No intraoperative and neither postoperative complications occurred (Clavien-Dindo 0). Hospital duration was 2 days. Preoperative eGFR was $112.0 \mathrm{~mL} / \mathrm{min} / 1.73 \mathrm{~m}^{2}$ and postoperative 1 -week eGFR was $105.7 \mathrm{~mL} / \mathrm{min} / 1.73 \mathrm{~m}^{2}$. The pathology result revealed pT1aN0M0 clear cell renal cell carcinoma (ISUP grade III) with a negative surgical margin (Table 2).

\section{Case 3}

A 38-year-old man diagnosed with a 4.6-cm hypervascular renal mass in the right upper-pole kidney (Figure 1C). The patient was obese with BMI of $33.86 \mathrm{~kg} / \mathrm{m}^{2}$ (Table 1). There was conversion to multiport retroperitoneal robot-assisted partial nephrectomy (RPN) due to limited retroperitoneal space by obesity and upper pole location renal mass. In case of upper pole renal mass, kidney elevation and traction were restricted due to flexible instruments. Therefore, left-side instrument was converted to multiport bipolar fenestrate forceps. Additionally, tumor resection was challenging due to lack of Endowrist of cold cup scissors, therefore the right-side instrument was also converted to multiport monopolar scissors. Total operative time was 248 minutes. The estimated blood loss was $550 \mathrm{~mL}$. The warm ischemia time was 55 minutes due to conversion to multiport conversion. Hospital duration was 7 days. Preoperative eGFR was $76.8 \mathrm{~mL} / \mathrm{min} / 1.73 \mathrm{~m}^{2}$ and postoperative 1-week eGFR was $54.0 \mathrm{~mL} / \mathrm{min} / 1.73 \mathrm{~m}^{2}$. There was Clavien-Dindo 1 complication. Oncological outcome revealed that the pathology result revealed pT1aN0M0 clear cell renal cell carcinoma (ISUP grade III) with a negative surgical margin (Table 2).

\section{Discussion}

The patient was placed in the full lateral position and the table was fully flexed to increase the space between the 12 th rib and iliac crest. This provides full access to the retroperitoneal space, and exposure of the whole abdomen in case of possible conversion to a transperitoneal approach. The spine and hip should be in a straight line, and the spine was fully exposed to allow space for the lateral robotic arm. Elbows should be placed forwards, so that the robotic arms do not collide with the patient's arms. To identify placement of the single site port, the iliac crest, ribs and axillary lines have been marked. If the camera port is too close to the 12 th rib, this may cause the robotic instruments to be too close to the kidney. For the single-site retroperitoneal approach, the incision was $3 \mathrm{~cm}$, which two fingers above the iliac crest just lateral to the triangle of Petit (Figure 2A). The external oblique muscle was separated using retractors to expose the lumbo-dorsal fascia (Figure 2B). A Kelly forceps was used to enter the retroperitoneal space. An index finger was then inserted into this space. The surgeon felt the tip of the 12th rib and the psoas muscle. A PDB balloon dilator (Tyco, Princeton, NJ, USA) was placed into the retroperitoneal space (Figures 2C,3A). Full dilation of retroperitoneal space was made allowing for the insertion of the Alexis retractor component of the "Lapsingle Vision" advanced access platform (Sejong Med, Paju, Korea) with four channels (Figure 3B). The channels were highly elastic. Therefore, the devices of various sizes ranging from 3 to $15 \mathrm{~mm}$ were applied. The 12 o'clock channel for the laparoscopic camera was placed toward the kidney. The remained two channels (3, 9 o'clock) were used for the working instruments, and the camera channel with the working instruments were positioned in a triangular fashion. The $0^{\circ} 8.5$-mm da Vinci stereo-laparoscope was connected to the robotic camera system and then the patient cart was positioned at the patient's posterior side cart and docked in a direction compatible with longitudinal axis of the kidney as anteroposterior configuration. Then, the two 5-mm curved cannulas were inserted into the docked robotic arms, and the semi-rigid instruments were loaded. Flexible robotic instruments (a monopolar hook and fenestrated bipolar forceps) were inserted through the curved cannulas (Figure 3C,D). The da Vinci system software automatically re-associates the surgeon's hands 
Table 2 Perioperative and postoperative outcomes of patients

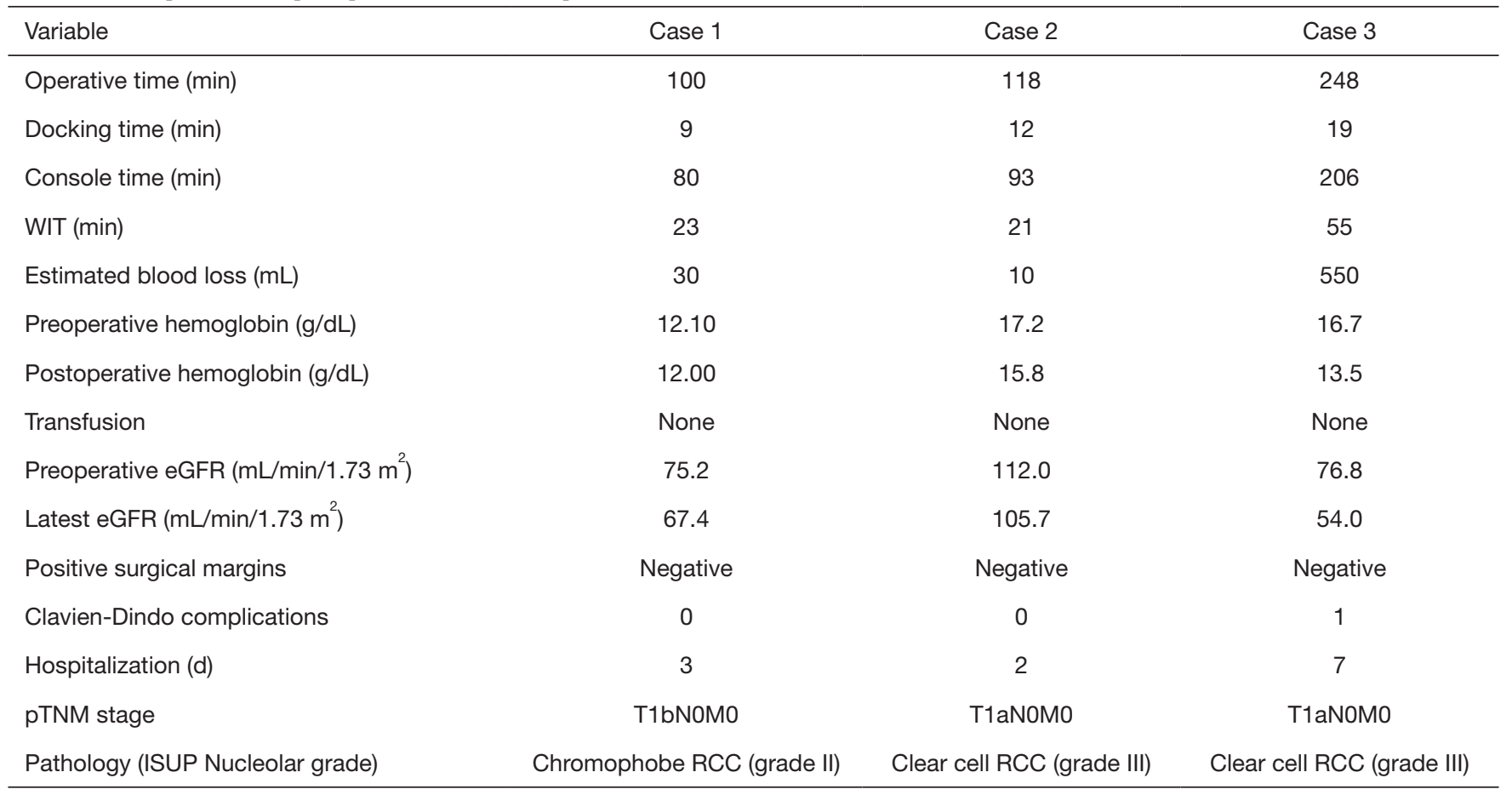

WIT, warm ischemic time; eGFR, estimated glomerular filtration rate; RCC, renal cell carcinoma.
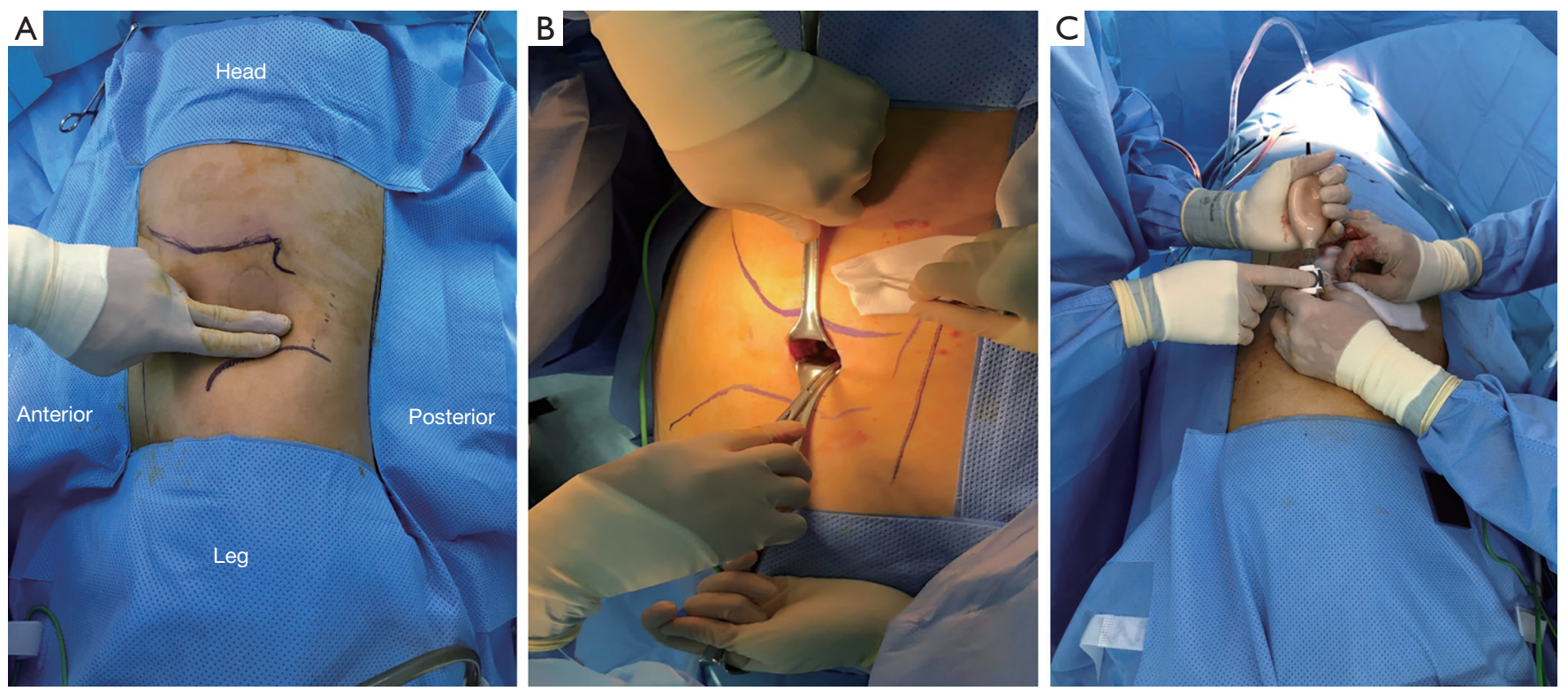

Figure 2 Retroperitoneal space creation. (A) The incision was $3 \mathrm{~cm}$, which was two fingers above the iliac crest just lateral to the triangle of Petit; (B) the external oblique muscle was separated using retractors to expose the lumbo-dorsal fascia; (C) a PDB balloon dilator (Tyco, Princeton, NJ, USA) was placed into the retroperitoneal space. 

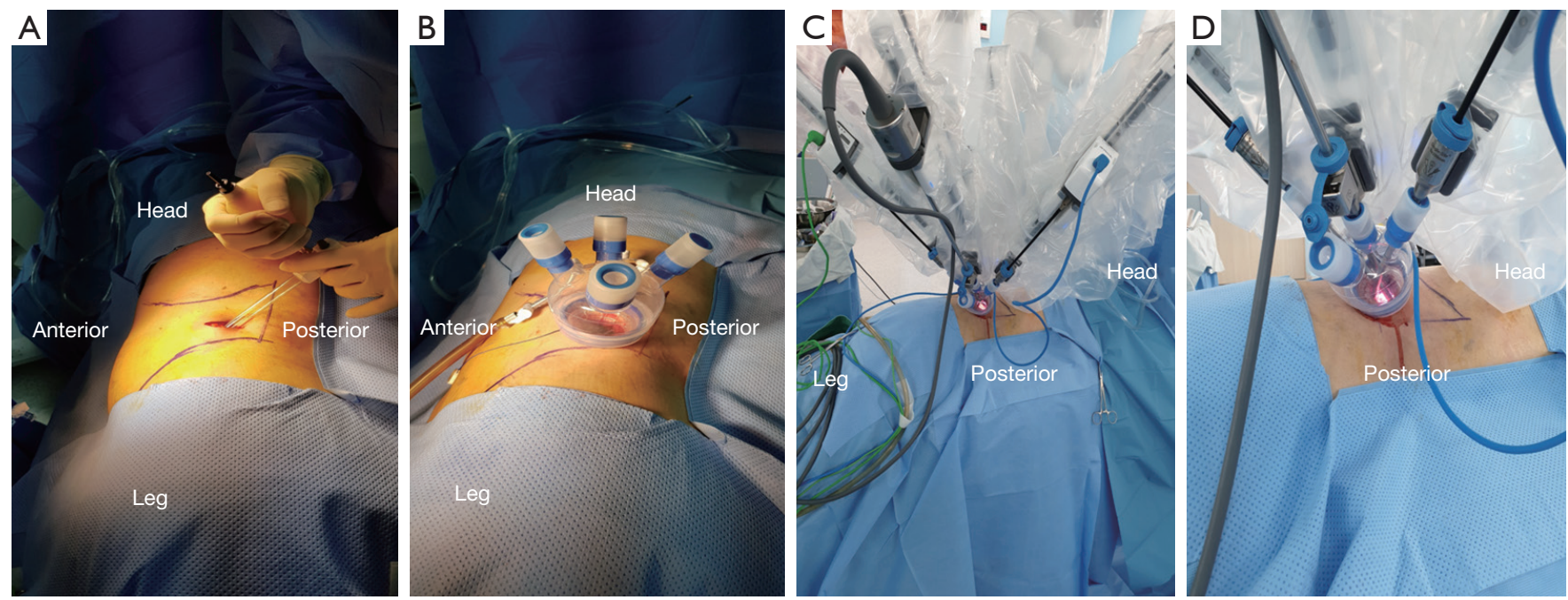

Figure 3 The port placement and docking procedure of retroperitoneal single-site robotic surgery using Lapsingle Vision advanced assess platform. (A) After retroperitoneal space creation; (B) lap single vision configuration for left side retroperitoneal single-site robot-assisted partial nephrectomy; (C,D) robotic arm installation and docking of robot during retroperitoneal surgery. Robot is docked over the patient's head, parallel to the spine.

with the instrument tips after docking, so that the left hand of the surgeon controls the right arm of the robot, and vice versa. Inside the retroperitoneal space, ureteral peristalsis was identified (Figure 4A). The renal hilum was isolated and prepared for clamping (Figure $4 B$ ). Defatting of the kidney was performed, the renal lesion was identified, and tumor boundaries were confirmed. The resection margin was scored with monopolar cautery using the robotic hook (Figure $4 C$ ). Clamping of the renal artery was obtained using bulldog clamps (Aesculap, Tuttlingen, Germany) (Figure $4 D$ ). Tumor enucleation/resection was performed by combining blunt and sharp dissection (Figure 4E). Renorrhaphy was performed in a double layer, reproducing the "sliding-clip" technique (Figure 4F) (9). During the renorrhaphy phase, bed suturing of the tumor resection site was conducted with a continuous running suture, using about $15 \mathrm{~cm}$ of 3-0 V-Loc (Covidien, Dublin, Ireland) barbed suture material. The outer layer was repaired using one running 2-0 V-Loc sutures. Once the renorrhaphy was completed, the hilar occlusion bulldog clamps were removed (Video 1, surgical video of case 1).

Robot-assisted, multiport, partial nephrectomy is a minimally invasive technique that has been accepted worldwide for the treatment of small and medium-sized renal masses (9). With increasing experience of robotic surgeries, there has been a dramatic increase in the adoption of LESS in urology, because LESS achieves cosmetic outcomes superior to those of the multiport technique (10-12). Nevertheless, the initial trial of R-LESS partial nephrectomy technique was challenging and the advantages of this procedure had not been clearly demonstrated. Therefore, the single-site robotic platforms have been developed to address these challenges (13). In 2010, Intuitive Surgical Inc. addressed the problem of instrument collision by developing the da Vinci single-site surgical platform, using a novel set of single-site instruments and accessories specifically dedicated for robot-assisted singlesite surgery. With this instrumentation, extracorporeal robotic arm clashing is minimized externally owing to the curved cannulas that angle the robotic arms far away from one another. Internal collision with the camera was avoided because the camera was designed to be placed in the middle of the curved cannula. Compared to multiport robotic system, the R-LESS system lacked various instruments, and there is no Endowrist technology in the R-LESS platform. Nevertheless, the technical advantages of the robotic platform made R-LESS more feasible than the conventional laparoscopic platform. This novel set of single-site instruments and accessories is specifically dedicated to R-LESS to overcome the current limitations. However, the assistant port had limited size and limitation of motion range. The docking is simpler, guided by a boommounted architecture with laser. Nevertheless, until now, there had been no cases of retroperitoneal approach single- 

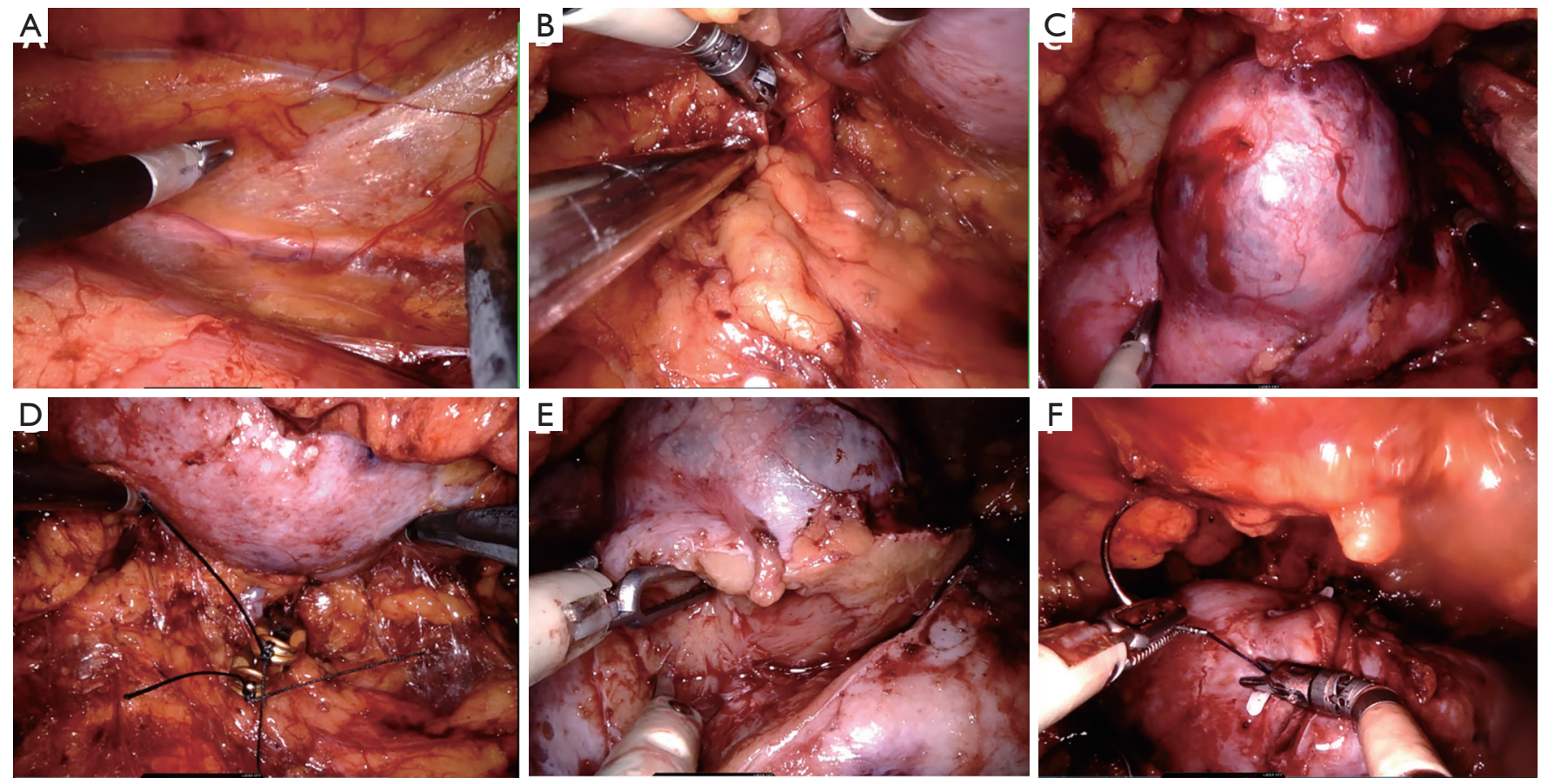

Figure 4 The step by step surgical procedure of retroperitoneal single-site robot-assisted partial nephrectomy. (A) Retroperitoneal anatomic relationships. The robotic scope is rotated for the psoas muscle in horizontal position. The ureter peristalsis is identified after balloon dissection of the retroperitoneum; (B) arterial pulsations are helpful to identify the renal artery, which is skeletonized in anticipation of hilar vascular control; (C) planning the excision margins by allowing accurate identification of the location, depth, and borders of the tumor; (D) hilar clamping can be performed using laparoscopic bulldog clamps; (E) the tumor is resected along the previously scored margin using cold scissors; (F) renorrhaphy is performed in two layers using robotic needle-drivers.

site partial nephrectomy performed using the Xi platform surgical system. Although the retroperitoneal approach was first described by Gaur et al. in 1993 (14), there has been relatively less utilization compared with transperitoneal approaches. This could be caused by larger working space and more familiar anatomic landmarks afforded by transperitoneal approach. The retroperitoneal approach is limited by a smaller working space, and the absence of anatomic landmarks which may disorient the surgeon and create the risk of inadvertent vascular injury requiring open conversion (15). Also, using a retroperitoneal approach might be more challenging, because of creation of the retroperitoneal space is a critical step and has to be performed carefully to avoid injury to the peritoneum. If peritoneum damage occurs during the process of making the retroperitoneal space, surgery can become very difficult. Furthermore, it is burdensome to make the retroperitoneal space and might require multiport RPN. However, the advantage of retroperitoneal approach is that access to the renal hilum is quick and relatively easy compared to transperitoneal approach. Often, the renal artery is encountered first. As a result, renal hilum dissection is completed faster. Because of the advantage of fast renal hilum access, the retroperitoneal approach might translate into shorter operation time.

In this report, we demonstrated the feasibility and effectiveness of pure single-site retroperitoneal RPN by da Vinci Xi robotic system using the specifically designed single-site platform for R-LESS. We had used "Lapsingle Vision" (Sejong Med, Paju, Korea) in combination with wound protectors through which robotic instruments were successfully placed instead of Intuitive robotic multichannel port (Intuitive Surgical, Sunnyvale, CA, USA). The first multichannel port (Intuitive Surgical, Sunnyvale, CA, USA) for single-site robot platform could not handle an assist port over $8 \mathrm{~mm}$. This issue with additional assist port over 
$10 \mathrm{~mm}$ meant R-LESS was not a "pure" single-site surgery. Nevertheless, the modified port made by "Lapsingle Vision" could achieve pure single-site surgery.

We overcame these disadvantages issues using modified port using "Lapsingle Vision" that rearranged the trocar site intra-operatively. The major advantage of da Vinci $\mathrm{Xi}$ compared to da-Vinci SP in retroperitoneal single-site $\mathrm{RPN}$ is intra-operative rearrangement of trocar location. Therefore, the relocation of robotic arm could make retroperitoneal single-site RPN tolerable in spite of limited retroperitoneal space.

Even if the feasibility and safety of R-SSRPN, there are several limitations of this technique.

In the cases of upper pole renal mass or with high BMI patient, this technique has difficulty to proceed on surgery due to restricted retroperitoneal space and difficulty of using flexible instruments. Therefore, careful patient selection is mandatory to perform R-SSRPN.

\section{Conclusions}

In conclusion, this initial case series with R-SSRPN by da Vinci Xi surgical system using Lapsingle Vision demonstrated that it is safe and effective treatment modality except for upper pole renal mass with high BMI patient. Further studies are needed to validate our results.

\section{Acknowledgments}

Funding: This research was supported by the National Research Foundation of Korea (NRF) grant funded by the Korea government (MSIT) (NRF-2017R1C1B5018097) and Basic Science Research Program through the National Research Foundation of Korea (NRF) funded by the Ministry of Education (NRF-2018R1D1A1B07051225).

\section{Footnote}

Conflicts of Interest: All authors have completed the ICMJE uniform disclosure form (available at http://dx.doi. org/10.21037/tau.2020.01.14). KHR serves as an unpaid editorial board member of Translational Andrology and Urology from Jun 2019 to May 2021. The other authors have no conflicts of interest to declare.

Ethical Statement: The authors are accountable for all aspects of the work in ensuring that questions related to the accuracy or integrity of any part of the work are appropriately investigated and resolved. Written informed consent was obtained from the patients for publication of this case report and accompanying images.

Open Access Statement: This is an Open Access article distributed in accordance with the Creative Commons Attribution-NonCommercial-NoDerivs 4.0 International License (CC BY-NC-ND 4.0), which permits the noncommercial replication and distribution of the article with the strict proviso that no changes or edits are made and the original work is properly cited (including links to both the formal publication through the relevant DOI and the license). See: https://creativecommons.org/licenses/by-nc$\mathrm{nd} / 4.0 /$.

\section{References}

1. Choi KH, Ham WS, Rha KH, et al. Laparoendoscopic single-site surgeries: a single-center experience of 171 consecutive cases. Korean J Urol 2011;52:31-8.

2. Shin TY, Lim SK, Komninos C, et al. Laparoendoscopic single-site (LESS) robot-assisted partial nephrectomy (RAPN) reduces postoperative wound pain without a rise in complication rates. BJU Int 2014;114:555-61.

3. Kallingal GJ, Swain S, Darwiche F, et al. Robotic Partial Nephrectomy with the Da Vinci Xi. Adv Urol 2016;2016:9675095.

4. Haber GP, White MA, Autorino R, et al. Novel robotic da Vinci instruments for laparoendoscopic single-site surgery. Urology 2010;76:1279-82.

5. Sorokin I, Canvasser NE, Irwin B, et al. The Decline of Laparoendoscopic Single-Site Surgery: A Survey of the Endourological Society to Identify Shortcomings and Guidance for Future Directions. J Endourol 2017;31:1049-55.

6. Cestari A, Buffi NM, Lista G, et al. Feasibility and preliminary clinical outcomes of robotic laparoendoscopic single-site (R-LESS) pyeloplasty using a new single-port platform. Eur Urol 2012;62:175-9.

7. Mathieu R, Verhoest G, Vincendeau S, et al. Roboticassisted laparoendoscopic single-site radical nephrectomy: first experience with the novel Da Vinci single-site platform. World J Urol 2014;32:273-6.

8. Paulucci DJ, Abaza R, Eun DD, et al. Robot-assisted partial nephrectomy: continued refinement of outcomes beyond the initial learning curve. BJU Int 2017;119:748-54.

9. Aboumarzouk OM, Stein RJ, Eyraud R, et al. Robotic versus laparoscopic partial nephrectomy: a systematic 
review and meta-analysis. Eur Urol 2012;62:1023-33.

10. Kaouk JH, Autorino R, Kim FJ, et al. Laparoendoscopic single-site surgery in urology: worldwide multiinstitutional analysis of 1076 cases. Eur Urol 2011;60:998-1005.

11. Tiu A, Shin TY, Kim KH, et al. Robotic laparoendoscopic single-site transumbilical partial nephrectomy: functional and oncologic outcomes at 2 years. Urology 2013;82:595-9.

12. Park SK, Olweny EO, Best SL, et al. Patient-reported body image and cosmesis outcomes following kidney surgery: comparison of laparoendoscopic single-site,

Cite this article as: Lee HH, Yoon YE, Kim YS, Na JC, Rha KH, Han WK, Kim DK. Retroperitoneal single-site robotassisted partial nephrectomy using Lapsingle Vision advanced access platform: initial three case reports. Transl Androl Urol 2020;9(2):758-765. doi: 10.21037/tau.2020.01.14 laparoscopic, and open surgery. Eur Urol 2011;60:1097-104.

13. Kaouk JH, Autorino R, Laydner H, et al. Robotic singlesite kidney surgery: evaluation of second-generation instruments in a cadaver model. Urology 2012;79:975-9.

14. Gaur DD, Agarwal DK, Purohit KC. Retroperitoneal laparoscopic nephrectomy: initial case report. J Urol 1993;149:103-5.

15. McAllister M, Bhayani SB, Ong A, et al. Vena caval transection during retroperitoneoscopic nephrectomy: report of the complication and review of the literature. J Urol 2004;172:183-5. 